DAMTP/97-50

hep-th/9712195

\title{
Connections between M-theory and superstrings
}

\author{
Michael B. Green \\ Department of Applied Mathematics and Theoretical Physics, Silver Street, \\ Cambridge CB3 9EW, United Kingdom \\ M.B.Green@damtp.cam.ac.uk
}

\begin{abstract}
:
This article reviews the non-perturbative structure of certain higher derivative terms in the type II string theory effective action and their connection to one-loop effects in eleven-dimensional supergravity compactified on a torus. New material is also included that was not presented in the talks. 10
\end{abstract}

\footnotetext{
${ }^{1}$ Based on talks given at the ICTP Conference on Duality Symmetries in String Theory, 7-12 April 1997; STRINGS '97, Amsterdam, June 16-21 1997; CERN Workshop on Nonperturbative Aspects of Strings, Branes and Fields, 8-12 December 1997.
} 


\section{Introduction}

Many features of M-theory follow directly from the strong algebraic constraints associated with the symmetries of the theory rather than specific details of any microscopic model, such as the matrix model. Certain of these properties must be inevitable consequences of eleven-dimensional supersymmetry. Others may depend on assuming the validity of the intricate web of duality inter-relationships between the perturbative string theories. Presumably a microscopic description, perhaps based on the matrix model, is needed to define the theory on all length scales - indeed, to define the very notion of a length scale. But it is of interest to determine the extent to which terms in a lowmomentum expansion of the M-theory effective action are determined simply by eleven-dimensional supergravity compactified in various ways.

It has been generally assumed that since perturbative supergravity is terribly divergent in eleven dimensions no interesting consequences follow by considering its loop diagrams. However, this ignores the powerful supersymmetry constraints which are expected to reduce the number of arbitrary constants but by how much? Furthermore, the eleven-dimensional quantum theory compactified to ten or fewer dimensions is supposed to be precisely equivalent to string theory and its compactifications. What mileage can be gained by exploiting this equivalence as an ansatz? The lowest order terms in the expansion of the M-theory action in powers of the momentum are the terms in the original classical supergravity action of [1]. Certain higher order terms have also been unambigously identified. One example is the $C^{(3)} \wedge X_{8}$ term [2] that arises as a one-loop effect in type IIA string theory [3] and can also be motivated by requiring the cancellation of chiral anomalies in $p$-branes 伅. Here $C^{(3)}$ is the Ramond-Ramond three-form and $X_{8}$ is an eight-form made out of the Riemann curvature. The presence of this term in the effective action, together with supersymmetry, must imply the presence of an infinite number of other terms. Some of these terms will be the subject of this article.

In the next section I will review the evidence that certain 'protected' terms 
in the low energy effective type II string action compactified on $S^{1}$ are precisely determined by one-loop effects in eleven-dimensional supergravity compactified on $T^{2}$. For example the one-loop eleven-dimensional four-graviton amplitude leads to a $R^{4}$ term in M-theory 1 which is related by supersymmetry to the $C^{(3)} \wedge X_{8}$ term. This term can be expressed in the type IIB coordinates by making use of the equivalence of M-theory compactified on $T^{2}$ with type IIB string theory compactified on $S^{1}$ [5, 6]. This leads to a term of the form $f(\rho, \bar{\rho}) R^{4}$ term in the type IIB action [7] where $f$ is a $S L(2, Z)$-invariant function of the complex IIB scalar field, $\rho$. The modular properties of the IIB theory are inhereted from the geometric $S L(2, Z)$ of $T^{2}$ in M-theory with $\rho$ identified with the complex structure, $\Omega$, of the torus. The interplay between the duality symmetries of nine-dimensional type II string theories and the one-loop eleven-dimensional supergravity amplitude is encoded in $f$ which contains both perturbative terms and non-perturbative D-instanton contributions. Furthermore, even though the eleven-dimensional loop amplitude is cubically divergent the consistent identification of the compactified theory with the IIB theory determines a specific finite renormalized value for the coefficient of the $R^{4}$ term in eleven dimensions - this finite value would necessarily arise as the regularized value in any microscopic theory. In fact, since the ultraviolet divergence is proportional to the volume of the compactification torus, it disappears in the zero-volume limit, and the exact (non-perturbative) $R^{4}$ term of ten-dimensional type IIB [8] is reproduced by finite terms alone.

The expression for the modular function $f(\rho, \bar{\rho})$ in the IIB theory will be motivated in section 2.1 by matching the known $R^{4}$ terms that arise at tree level and one loop in string perturbation theory together with the expected structure of multiply-charged D-instanton contributions. A single D-instanton of charge $N=\hat{m} n$ can be identified, following T-duality, with configurations in the IIA theory in which the world-line of a charge- $n$ D-particle winds $\hat{m}$ times around a compact dimension. Precisely the same expression will be obtained in section 2.2 from the low momentum limit of the one-loop four graviton scattering amplitude in eleven-dimensional supergravity compactified on $T^{2}$. I will also make some

\footnotetext{
${ }^{2}$ The notation $R^{4}$ represents a particular contraction of four Riemann curvature tensors that will be reviewed below.
} 
comments concerning the relation of the higher-order terms in the momentum expansion of the one-loop supergravity amplitude with string loop diagrams (related comments are made in [9]).

The $R^{4}$ term is related by supersymmetry to a large number of other terms. In the language of the type IIB theory these terms include a sixteen-fermion term, $f_{16}(\rho, \bar{\rho}) \lambda^{16}$, where $\lambda$ is the complex spin- $\frac{1}{2}$ chiral fermion [10]. This is the analogue of the 't Hooft fermion vertex in a conventional Yang-Mills instanton background. These interaction terms can be expressed as integrals over half the ten-dimensional on-shell superspace (there is no ten-dimensional off-shell superspace formalism). Such protected terms again only receive perturbative contributions at tree-level and at one loop in string theory and appear to be determined entirely by one-loop diagrams in eleven-dimensional supergravity. A brief overview of such terms is given in section 2.3.

The expressions for the instanton contributions in the expansions of these interaction terms defines a measure on the space of charge- $N$ D-instantons. In [11 it was argued that this should equal the partition function of the zerodimensional Yang-Mills model, which is an integral over bosonic and fermionic matrices in the Lie algebra of $S U(N)$ [12]. This, in turn, is related to the bulk term in the Witten index for charge- $N$ D-particles [13, 14]. This circle of arguments which is presented in detail in [11] will be briefly reviewed at the end of section 2.3 .

It would be very interesting to discover how much of the structure of the string perturbation expansion can be determined from eleven-dimensional supergravity beyond these protected terms. For example, the $R^{4}$ term is the lowest-order term in a momentum expansion of the exact four-graviton scattering amplitude in type IIB string theory. There are very likely to be terms of higher order in momenta that are also protected by supersymmetry. To what extent can the momentum expansion of the effective action be determined by perturbation theory in eleven dimensions? A more limited question is to what extent the momentum expansion of the string tree-level amplitude can be reproduced by eleven-dimensional supergravity perturbation theory? This depends on the systematics of the multi-loop diagrams in eleven-dimensional supergravity as will be described in section 3. These diagrams have finite pieces that 
depend on the moduli of the compact dimensions. New primitive divergences arise corresponding to terms which have derivatives acting on $R^{4}$, denoted symbolically as $\partial^{2 n} R^{4}$. It would be interesting to see whether these might be determined by the same kind of arguments that led at one loop to the determination of the $R^{4}$ term. However, the arguments that I present beyond one loop are based on dimensional analysis rather than explicit evaluation of the rather complicated Feynman diagrams. In this way it is easy to see how specific multi-loop diagrams could give specific finite terms in the M-theory action that correspond to terms that arise from string perturbation theory. However, it seems likely that the values of the counterterms are fixed unambiguously only at low orders in the momentum expansion where they are protected by supersymmetry.

\section{Higher-order terms in M-theory}

\section{1 $S L(2, Z)$-invariant $R^{4}$ terms in type IIB}

The leading perturbative contributions to the four graviton scattering amplitude in type IIB superstring theory are of the form (in string frame)

$$
S_{R^{4}}^{\text {pert }}=\int \sqrt{g^{B}}\left(\zeta(3) \rho_{2}^{2}+\frac{\pi^{2}}{3}\right) t^{8} t^{8} R^{4},
$$

where $g_{\mu \nu}^{B}$ is the IIB string-frame metric and

$$
t_{8} t_{8} R^{4} \equiv t^{\mu_{1} \ldots \mu_{8}} t_{\nu_{1} \ldots \nu_{8}} R_{\mu_{1} \mu_{2}}^{\nu_{1} \nu_{2}} \cdots R_{\mu_{7} \mu_{8}}^{\nu_{7} \nu_{8}}
$$

where the rank-eight tensor, $t^{8}$, is defined in 15]. The first term in (1) is the treelevel contribution [16, 17] that is of order $\alpha^{\prime 3}$ relative to the leading EinsteinHilbert term. The second term is the one-loop contribution [18 and has no dependence on the dilaton in the string frame. Non-perturbative contributions to the $t_{8} t_{8} R^{4}$ term also arise from single D-instantons with charge $N$ [ $]$ which give an infinite series of non-perturbative contributions that have the form

$$
S_{R^{4}}^{\text {nonpert }}=\sum_{N>0} c_{N}\left(\rho_{2}\right)\left(e^{2 \pi i N \rho}+e^{-2 \pi i N \bar{\rho}}\right) t_{8} t_{8} R^{4} .
$$

One way of counting these D-instantons makes use of T-duality between the IIB and the IIA theories in nine dimensions. From the IIA point of view 
a D-instanton is associated with the world-line of a D-particle of charge $n$ and mass $e^{-\phi^{A}} n$ (where $\phi^{A}$ is the IIA dilaton) winding around the compact ninth dimension of radius $r_{A}$. The euclidean action of this configuration is $S=2 \pi \hat{m} n\left(C^{(1)}+i r_{A} e^{\phi^{A}}\right)$ where $C^{(1)}$ is the IIA one-form and $\hat{m}$ is the winding number of the world-line. After T-duality this leads to the expression $S=2 \pi N \rho$ for the D-instanton action where $N=\hat{m} n$. Although the coefficients $c_{N}$ are probably very hard to determine directly they are fixed by the requirement that the total action should be invariant under $S L(2, Z)$ transformations. This means that it must have the form,

$$
S_{R^{4}} \equiv S_{R^{4}}^{\text {pert }}+S_{R^{4}}^{\text {nonpert }}=\int \sqrt{g^{B}} \rho_{2}^{1 / 2} f(\rho, \bar{\rho}) t_{8} t_{8} R^{4} d^{10} x,
$$

where $f(\rho, \bar{\rho})$ is a modular function since $R$ is invariant under $S L(2, Z)$ transformations in the Einstein frame.

The precise coefficients of the known perturbative contributions, $S^{\text {pert }}$, together with the general form of the instanton corrections motivates the suggestion [8] that $f$ is given by

$$
f(\rho, \bar{\rho})=\pi^{-2} \sum_{\left(\hat{l}_{1}, \hat{l}_{2}\right) \neq(0,0)} \frac{\rho_{2}^{3 / 2}}{\left|\hat{l}_{1}+\rho \hat{l}_{2}\right|^{3}}=\pi^{-2} \zeta(3) E_{\frac{3}{2}}(\rho, \bar{\rho}),
$$

where the nonholomorphic Eisenstein series $E_{s}$ is defined by [19]

$$
\begin{aligned}
\zeta(2 s) E_{s}(\rho, \bar{\rho}) & =\sum_{\left(\hat{l}_{1}, \hat{l}_{2}\right) \neq(0,0)} \frac{\rho_{2}^{s}}{\left|\hat{l}_{1}+\rho \hat{l}_{2}\right|^{2 s}} \\
& =\zeta(2 s) \rho_{2}^{s}+\frac{\sqrt{\pi} \Gamma\left(s-\frac{1}{2}\right) \zeta(2 s-1)}{\Gamma(s)} \rho_{2}^{1-s}+\mathcal{R}_{s} .
\end{aligned}
$$

The last equality is a large- $\rho_{2}$ expansion and $\mathcal{R}_{s}$ indicates a specific sum of exponentially decreasing terms. In the special case $s=3 / 2$ this sum is given by

$$
\mathcal{R}_{\frac{3}{2}}=\sum_{N>0}\left(\sum_{N \mid \hat{m}} \frac{1}{\hat{m}^{2}}\right) N^{\frac{1}{2}}\left(e^{2 \pi i N \rho}+e^{-2 \pi i N \bar{\rho}}\right) \times \sum_{k=0}^{\infty}\left(2 \pi N \rho_{2}\right)^{-k} \frac{\Gamma\left(\frac{1}{2}-k\right)}{\Gamma\left(-\frac{1}{2}-k\right)},
$$

where $\sum_{N \mid \hat{m}}$ indicates a sum over the divisors of $N$. There are only two powerbehaved terms in the expansion (6) and they correspond precisely to the known tree-level and one-loop terms in the $R^{4}$ effective action of the IIB theory, while 
the series of exponentials in (7) correspond to D-instanton corrections with the expected instanton number $N=\hat{m} n$. This lends weight to the suggestion that (5) is the exact result, in which case there should be a perturbative nonrenormalization theorem [8] that forbids corrections to the $R^{4}$ term beyond one loop. Recently there has been a certain amount of evidence for the validity of such a theorem 20, 21] (although an apparent contradiction in the literature 22 deserves closer analysis). The measure factor $\sum_{N \mid n} \frac{1}{n^{2}}$ in the instanton sum (17) can be related [11] to the expression for the Witten index of relevance in the analysis of D-particle threshold bound states 13] [14 as will be described at the end of section 2.3 .

\subsection{One loop in eleven dimensions}

The leading low energy behaviour of the one-loop four-graviton amplitude of eleven-dimensional supergravity compactified on a torus with radii $R_{10}$ and $R_{11}$ was considered in [7]. Here we will consider the complete momentum dependence of the same amplitude (a similar argument was also given in [9) and expand the expression in a power series in the Mandelstam invariants, $S, T$ and $U$,

$$
S=-\left(k_{1}+k_{2}\right)^{2}, \quad T=-\left(k_{1}+k_{4}\right)^{2}, \quad U=-\left(k_{1}+k_{3}\right)^{2},
$$

so that $\mathrm{S}+\mathrm{T}+\mathrm{U}=0$.

The terms of interest arise from the sum of all one-loop diagrams with four external gravitons and with the graviton, gravitino or antisymmetric three-form potential circulating around the loop. This sum is most succinctly calculated in a first-quantized light-cone gauge formalism in which the amplitude is described as a trace over the states of an eleven-dimensional super-particle circulating around the loop and coupled to the four external gravitons by vertex operators (as in [7]). The result is given by

$$
A_{4}=\frac{1}{\kappa_{11}^{2}} \tilde{K}[I(S, T)+I(S, U)+I(U, T)],
$$

where $\tilde{K}$ is the linearized approximation to $R^{4}$ (which is eighth order in momenta and symmetric under the interchange of any pair of gravitons) and the function $I(S, T)$ has the form of a Feynman integral for a massless scalar field theory. It 
is given by

$$
I(S, T)=\frac{1}{\pi^{5 / 2} \mathcal{V}_{2}} \int \prod_{r=1}^{4} d \tau_{r} \int d^{9} q \sum_{\left\{l_{1}, l_{2}\right\}} e^{-G^{I J} l_{I} l_{J} \tau-\sum_{r=1}^{4} p_{r}^{2} \tau_{r}},
$$

where $\tau=\sum_{r=1}^{4} \tau_{r}$ and $q_{i}(i=1, \cdots, 9)$ is the nine-dimensional loop momentum transverse to $T^{2}$. The parameters $\tau_{i}$ label the relative positions of the four vertices. The sum is over the Kaluza-Klein momenta $\left(l_{1}\right.$ and $\left.l_{2}\right)$ in the two compact dimensions $(I, J=1,2)$ and the momenta in the legs of the loop are given by

$$
p_{r}=q+\sum_{s=1}^{r} k_{s}
$$

where the external momenta, $k_{r}^{\mu}$ satisfy $k_{r}^{2}=0$ and $\sum_{r=1}^{4} k_{r}=0$. The particular kinematic configuration has been chosen in which the external momenta have zero components in the directions of the torus, i.e. $k_{r}^{I}=0$. The elevendimensional coupling constant, $\kappa_{11}$, has dimension, $(\text { length })^{9 / 2}$ and will be set equal to 1 in most of the following. The inverse metric on $T^{2}$ is defined by

$$
G^{I J} l_{I} l_{J}=\frac{1}{\mathcal{V}_{2} \Omega_{2}}\left|l_{1}+\Omega l_{2}\right|^{2},
$$

where $\mathcal{V}_{2}$ is the volume of the torus with complex structure, $\Omega=\Omega_{1}+i \Omega_{2}$ (where $\Omega_{1}$ is an angular parameter and $\Omega_{2}=R_{10} / R_{11}$ ). Setting all the $k_{r}=0$ in (10) gives the lowest-order result of [7]. The full amplitude (9) gives rise to terms in the M-theory effective action compactified on $T^{2}$ of the form

$$
S_{4}=\frac{1}{\kappa_{11}^{2}} \int \sqrt{G^{(9)}} \mathcal{V}_{2} h\left(\mathcal{V}_{2}, \Omega ; \partial^{2}\right) R^{4} d^{9} x
$$

The function $h$ is a modular function of $\Omega$ and its argument $\partial^{2}$ symbolically indicates derivatives acting on the fields in $R^{4}$, corresponding to the dependence of $A_{4}$ on the momenta $k_{r}$.

After completing a square in the exponent of (10) and then performing the shifted loop integral the expression becomes

$$
I(S, T)=\frac{\pi^{2}}{\mathcal{V}_{2}} \int \prod_{r=1}^{4} d \tau_{r} \tau^{-9 / 2} \sum_{\left\{l_{1}, l_{2}\right\}} e^{-G^{I J} l_{I} l_{J} \tau+\frac{1}{\tau}\left(S \tau_{1} \tau_{3}+T \tau_{2} \tau_{4}\right)} .
$$


This integral is to be evaluated in the region $S, T<0$ where it converges and then analytically continued to the physical region.

The momentum-independent terms in (14) can be isolated by writing

$$
I(S, T)=I_{0}+I^{\prime}(S, T),
$$

where

$$
I_{0} \equiv I(0,0)=\frac{\pi^{3 / 2}}{\mathcal{V}_{2}} \int_{0}^{\infty} d \tau \tau^{-3 / 2} \sum_{\left\{l_{1}, l_{2}\right\}} e^{-\pi G^{I J} l_{I} l_{J} \tau},
$$

which is the expression considered in [7]. It has a divergence in the limit $\tau=0$. A double Poisson resummation reexpresses $I_{0}$ as a sum over $\hat{l}_{1}$ and $\hat{l}_{2}$ that may be identified with the winding numbers of the euclidean world-line of the supergraviton around the directions $R_{11}$ and $R_{9}$ of the torus, respectively. The result is

$$
\begin{aligned}
I_{0} & =\pi^{3 / 2} \int_{0}^{\infty} d \tau \tau^{-5 / 2} \sum_{\left\{\hat{l}_{1}, \hat{l}_{2}\right\}} e^{-\pi G_{I J} \hat{l}_{I} \hat{l}_{J} \frac{1}{\tau}} \\
& =\pi^{3 / 2} \int_{0}^{\infty} d \hat{\tau} \hat{\tau}^{\frac{1}{2}} \sum_{\left\{\hat{l}_{1}, \hat{l}_{2}\right\}} e^{-\pi G_{I J} \hat{l}_{I} \hat{l}_{J} \hat{\tau}}
\end{aligned}
$$

where $\hat{\tau}=1 / \tau$. This isolates the divergence in the zero winding term $\left(\hat{l}_{1}=\hat{l}_{2}=\right.$ $0)$. This is presumably regularized by a microscopic theory, such as the matrix model [23], but its regularized value is also determined uniquely by requiring that $I_{0}$ reproduce the IIA and IIB string theory $R^{4}$ terms in nine dimensions. Since the loop diverges as $\Lambda^{3}$, where $\Lambda$ is a momentum cut-off, the regularized value of this term has the form $c \kappa_{11}^{-2 / 9}$ where $c$ is a dimensionless constant. The remaining terms in (17) depend on the volume and complex structure of $T^{2}$ and are finite. The $\hat{\tau}$ integral is trivial for these terms and (17) can be written as

$$
I_{0}=c \kappa_{11}^{-2 / 9}+\mathcal{V}_{2}^{-3 / 2} \zeta(3) E_{\frac{3}{2}}(\Omega, \bar{\Omega}) .
$$

Substituting into (15) and (9) leads to a $R^{4}$ contribution to the M-theory effective action that can be expressed in terms of the IIB theory compactified on a circle. 3

\footnotetext{
${ }^{3}$ This makes use of the usual relations between the parameters of M-theory on $T^{2}$ and the type II string theories on $S^{1}: r^{A}=\left(r^{B}\right)^{-1}=R_{10}\left(R_{11}\right)^{\frac{1}{2}}, e^{\phi^{B}}=\left(r^{A}\right)^{-1} e^{\phi^{A}}=R_{11} / R_{10}$, where $r^{A}$ is the radius of the IIA circle in string units and $\phi^{A}$ and $\phi^{B}$ are the dilatons of the two type II theories that are related by T-duality.
} 
In the limit $\mathcal{V}_{2} \rightarrow 0$ the radius, $r_{B}$, of the IIB circle becomes infinite and the second term in (18) dominates, leading to an expression for the action for the decompactified IIB theory which coincides with (雨) (with $f$ defined by (5)). The IIB string tree-level contributions arise from terms in (17) with $\hat{l}_{1} \neq 0$ and $\hat{l}_{2}=0$ so the loop has non-zero winding only in the eleventh dimension. The string one-loop and D-instanton terms in (画) can be extracted from the $\hat{l}_{2} \equiv \hat{m} \neq 0$ terms in (17) by performing a Poisson summation that takes the winding numbers $\hat{l}_{1}$ into Kaluza-Klein charges $n$ and identifying $N=\hat{m} n$. The $n=0$ term gives rise to the term in (柯) that is independent of the dilaton (the one-loop contribution) while the charge- $N$ D-instanton contributions in (7) come from the $N \neq 0$ contributions.

Although the zero winding number term with coefficient $c$ does not contribute in the $\mathcal{V}_{2} \rightarrow 0$ limit it does contribute to the finite- $\mathcal{V}_{2}$ amplitude. By transforming to the type IIA coordinates it is easy to see that $c$ is the coefficient of the one-loop contribution to the $R^{4}$ term in the IIA theory in ten dimensions and takes precisely the same value as the one-loop term in $S^{\text {pert }}$, thereby ensuring the T-duality of the type IIA and IIB theories [7].

There is an intriguing analogy between this calculation and the calculation of the exact prepotential of four-dimensional $N=2$ supersymmetric Yang-Mills theory from a one-loop amplitude in five dimensions [24.

The momentum-dependent terms in $I(S, T)$ in (15) are contained in $I^{\prime}(S, T)$. This is evaluated by separating the $l_{1}=l_{2}=0$ term from the rest,

$$
I^{\prime}(S, T)=I^{0}(S, T)+\sum_{n=2}^{\infty} I_{n}(S, T) .
$$

The term with zero Kaluza-Klein momenta, $I^{0}$, gives a contribution to the amplitude that is non-analytic in the Mandelstam invariants while $I_{n}$ is a homogeneous polynomial in $S$ and $T$ of degree $n$. After translating to string coordinates the term $I^{0}$ can be identified with a corresponding one-loop term in string perturbation theory. The polynomials $I_{n}(S, T), I_{n}(T, U)$ and $I_{n}(U, S)$ can be identified with terms that should arise from multi-loop amplitudes in the supergravity theory. More details are given in the Appendix which overlaps with 99. 
Compactification of the supergravity loop amplitude to eight dimensions on $T^{3}$ raises new issues associated with the presence of new instantons that are identified with wrappings of the M-theory membrane world-volume around $T^{3}$. The complete expression for the $R^{4}$ term is now obtained by combining the one-loop calculation with the constraints of U-duality [25, 4, 26]. Related issues arise in considerations of type I/heterotic duality in eight dimensions [27, 28]. Compactification to lower dimensions raises yet more issues analogous to those that arise in the compactification of the matrix model.

\subsection{Sixteen-fermion and related terms}

The $f(\rho, \bar{\rho}) t_{8} t_{8} R^{4}$ term is one of a large number of terms of the same dimension that are related to each other by supersymmetry. Prominent among these is the analogue of the 't Hooft multi-fermion vertex of the IIB theory,

$$
S_{\lambda^{16}}=\int \sqrt{g^{B}} \rho_{2}^{1 / 2} f_{16}(\rho, \bar{\rho}) \lambda^{16} d^{10} x,
$$

where $\lambda$ is the complex chiral spin- $\frac{1}{2}$ fermion. This field transforms with weight $3 / 2$ under the $U(1)$ denominator in the $\operatorname{coset} S L(2, Z) / U(1)$ [29, 30, 31] so that a general $S L(2, Z)$ transformation acts as

$$
\lambda \rightarrow\left(\frac{c \bar{\rho}+d}{c \rho+d}\right)^{\frac{3}{4}} \lambda
$$

As a consequence of $S L(2, Z)$ invariance of the action it follows that the coefficient $f_{16}$ in (20) is a non-holomorphic modular form of weight $(12,-12)$ (where the notation indicates the holomorphic and anti-holomorphic weights, respectively).

The expression for $f_{16}$ can also be obtained by a one-loop calculation in eleven-dimensional supergravity compactified on $T^{2}$. This time the process has sixteen external gravitini in polarization states that correspond to $\lambda$ in the IIB description. With this choice of external states the loop diagram vanishes in the limit of eleven non-compact dimensions and is finite for generic $T^{2}$. It was evaluated in a recent paper [10] and the result is

$$
f_{16}=\frac{1}{\pi^{2}} \rho_{2}^{3 / 2} \Gamma(27 / 2) \sum_{\left(\hat{l}_{1}, \hat{l}_{2}\right) \neq(0,0)} \frac{\left(\hat{l}_{1}+\hat{l}_{2} \bar{\rho}\right)^{24}}{\left|\hat{l}_{1}+\hat{l}_{2} \rho\right|^{27}},
$$


where $\hat{l}_{1}$ and $\hat{l}_{2}$ are the winding numbers of the world-line around the cycles of the torus. This expression has the large $\rho_{2}$ expansion,

$$
\begin{aligned}
\rho_{2}^{1 / 2} f_{16}= & \pi^{-2} \Gamma(27 / 2) \zeta(3)\left(\rho_{2}\right)^{2}+\pi^{-2} \Gamma(23 / 2) \zeta(2) \\
& +2^{24} \pi^{23 / 2} \sum_{N>0}\left(\sum_{N \mid \hat{m}} \frac{1}{\hat{m}^{2}}\right)\left(N \rho_{2}\right)^{25 / 2} e^{2 \pi i N \rho}\left(1+O\left(\rho_{2}^{-1}\right)\right),
\end{aligned}
$$

which again indicates the presence of string perturbation theory contributions at tree-level and one-loop together with an infinite number of D-instanton contributions (the anti D-instantons enter with coefficients that are of higher order in $\rho_{2}^{-1}$ ). There are presently no sixteen-fermion perturbative string calculations in the literature with which to compare the two leading terms.

In [10] it was also shown that the functions $f_{16}$ and $f$ are related by

$$
f_{16}(\rho, \bar{\rho})=\rho_{2}^{12} \mathcal{D}^{12} f(\rho, \bar{\rho}) .
$$

The covariant derivative $\mathcal{D}$ is defined by

$$
F_{d+2, \bar{d}}=\mathcal{D}_{d} F_{d, \bar{d}}=i\left(\frac{\partial}{\partial \rho}+\frac{d}{(\rho-\bar{\rho})}\right) F_{d, \bar{d}}
$$

where $F_{d, d^{\prime}}$ is a modular form of holomorphic weight $d$ and anti-holomorphic weight $d^{\prime}$ and

$$
\mathcal{D}^{k} F_{d, \bar{d}} \equiv \mathcal{D}_{d+2(k-1)} \mathcal{D}_{d+2(k-2)} \cdots \mathcal{D}_{d} F_{d, \bar{d}} .
$$

The relationship (24) between $f$ and $f_{16}$ is consistent with the consequences of linearized supersymmetry. The physical on-shell fields of linearized IIB supergravity are contained in a superfield $\Phi(x, \theta)$ (where $\theta$ is a complex chiral Grassmann spinor of $S O(9,1))$ satisfying the holomorphic constraint $D^{*} \Phi=0$ and the on-shell condition $D^{4} \Phi=D^{* 4} \Phi^{*}$ where $D$ and $D^{*}$ are supercovariant derivatives [31]. This field has an expansion in powers of $\theta$,

$$
\Phi=\rho_{0}+a-\frac{2 i}{g} \bar{\theta} \lambda-\cdots-\frac{i}{48 g} \bar{\theta} \Gamma^{\mu \nu \eta} \theta \bar{\theta} \Gamma_{\eta}{ }^{\sigma \tau} \theta R_{\mu \nu \sigma \tau}+\cdots,
$$

where $\rho_{0}=\rho-a$ is a constant background that defines the coupling constant in the linearized theory, $R$ is the linearized approximation to the curvature and ... indicates the other terms in the expansion. The matrices $\Gamma^{\mu}$ are $S O(9,1)$ 
gamma matrices and $\bar{\theta}=\Gamma^{0} \theta$ (with no complex conjugation). The linearized supersymmetric on-shell action has the form

$$
S^{\prime}=\operatorname{Re} \int d^{10} x d^{16} \theta g^{4} F[\Phi],
$$

and the various component interactions are contained in the $\theta^{16}$ term in the expansion of $F[\Phi]$. In addition to the $R^{4}$ and $\lambda^{16}$ terms there are many others some of these are singlets under the $U(1)$ (and arose at one string loop in 32]) while others carry a net even number of units of $U(1)$ charge 10] (some of these terms have also been discussed in [33]).

Although the fully nonlinear theory cannot easily be expressed in terms of $\Phi$ the leading behaviour of the charge- $N$ instanton term is obtained by substituting $F=N^{-7 / 2} e^{2 \pi i N \Phi} \sum_{N \mid n} \frac{1}{\hat{m}^{2}}$ into (28). In this manner the leading instanton contributions to the $R^{4}, \lambda^{16}$ and related terms are all contained in a simple superspace expression.

The expression for the measure on single charge- $N$ D-instantons, $\sum_{N \mid n} \frac{1}{\hat{m}^{2}}$, can be read off from the expressions for the instanton contributions to the various processes described above. This can then be compared with the expression that should follow from the matrix description of D-instantons. According to 12] this is determined by the partition function for $U(N)$ tendimensional supersymmetric Yang-Mills theory reduced to zero dimensions, in which case the configuration space consists of the ten $N \times N$ bosonic matrices, $A_{\mu}$, and sixteen $N \times N$ fermionic matrices, $\psi^{a}$. The partition function is given by

$$
Z_{U(N)}=\int d^{10} y d^{16} \epsilon Z_{S U(N)},
$$

where the center of mass super-translation zero modes have been separated from the measure factor,

$$
Z_{S U(N)}=\int_{S U(N)} D A D \psi \exp (-S(A, \psi)) .
$$

This integral, which enters into the matrix model of [34, has not been explicitly evaluated apart from the $N=2$ case considered in 13] 14. (which gave $Z_{S U(2)}=$ $5 / 4$ ). However, equating it with the measure for $N$ coincident D-instantons 
obtained from the $t_{8} t_{8} R^{4}$ and related terms strongly suggests that

$$
Z_{S U(N)}=\sum_{N \mid \hat{m}} \frac{1}{\hat{m}^{2}},
$$

for all $N$ [11]. It would obviously be of interest to check this by explicit evaluation of the finite-dimensional integral (30).

\section{Tree-level string theory and multi-loop super- gravity.}

We have seen that the lowest-dimensional terms in the string theory effective action correspond to terms that arise at one loop in eleven-dimensional supergravity. We would now like to see whether higher-order string-theory terms can be obtained from multi-loop amplitudes in eleven dimensions. To be explicit we will compare the expansion of the ten-dimensional IIA string tree amplitude with the compactification of multi-loop diagrams of eleven-dimensional supergravity on a circle of radius $R_{11}$. The calculation of multi-loop diagrams is obviously very difficult but we will be able to infer certain systematic properties from dimensional considerations.

First, consider the tree amplitude for four-graviton scattering in either of the type II superstring theories which is given by

$$
\begin{aligned}
A_{4}^{\text {tree }} & =e^{-2 \phi} \frac{\tilde{K}}{s t u} \frac{\Gamma\left(1-\alpha^{\prime} s\right) \Gamma\left(1-\alpha^{\prime} t\right) \Gamma\left(1-\alpha^{\prime} u\right)}{\Gamma\left(1+\alpha^{\prime} s\right) \Gamma\left(1+\alpha^{\prime} t\right) \Gamma\left(1+\alpha^{\prime} u\right)} \\
& =e^{-2 \phi} \frac{\tilde{K}}{s t u} \exp \left(\sum_{n=1}^{\infty} \frac{2 \zeta(2 n+1)}{2 n+1}\left(s^{2 n+1}+t^{2 n+1}+u^{2 n+1}\right)\right)
\end{aligned}
$$

where the string-frame Mandelstam invariants are related to those in the Mtheory coordinates by

$$
s=\frac{S}{R_{11}}, \quad t=\frac{T}{R_{11}}, \quad u=\frac{U}{R_{11}} .
$$

It is important that every term in the exponent can be expressed as a polynomial in $s$ and $t$ multiplied by $s t u$, as can be seen from the identity,

$$
s^{2 n+1}+t^{2 n+1}+u^{2 n+1}=s t u\left[\sum_{r=1}^{n} \sum_{q=0}^{2 n-2 r} \frac{(2 n+1) !}{r !(2 n+1-r) !}(-1)^{q} s^{2 n-1-r-q} t^{r+q-1}\right],
$$


where $n \geq 1$. This means that the massless poles only contribute to the first term in the expansion of the exponential.

When expressed in terms of the Mandelstam invariants in the M-theory metric the expression (32) has the low-energy expansion,

$$
\begin{aligned}
& A_{4}^{\text {tree }} \sim \tilde{K}\left(\frac{1}{S T U}+\frac{2 \zeta(3)}{R_{11}^{3}}+\frac{2 \zeta(5)}{R_{11}^{5}}\left(S^{2}+S T+T^{2}\right)+\frac{2 \zeta(3)^{2}}{R_{11}^{6}} S T U\right. \\
& +\frac{2 \zeta(7)}{R_{11}^{7}}\left(S^{4}+2 S^{3} T+3 S^{2} T^{2}+2 S T^{3}+T^{4}\right)+\frac{2 \zeta(3) \zeta(5)}{R_{11}^{8}} S T U\left(S^{2}+S T+T^{2}\right) \\
& \left.+\frac{2 \zeta(9)}{R_{11}^{9}}\left(S^{6}+4 S^{5} T+\cdots+T^{6}\right)+\frac{4}{3 R_{11}^{9}} \zeta(3)^{3} S^{2} T^{2} U^{2}+\cdots\right) .
\end{aligned}
$$

The first term in the expansion combines with the kinematic factor, $\tilde{K}$, to give the tree-level amplitude that is described by the Einstein-Hilbert action of eleven-dimensional supergravity compactified on a circle of radius $R_{11}=e^{2 \phi / 3}$. The subsequent term, with coefficient $\zeta(3)$ is the term considered earlier that comes from the $R^{4}$ term in the effective action. We saw that this term is reproduced by the one-loop diagram of eleven-dimensional supergravity compactified on a circle. The higher-order terms in (35) come from terms in the effective action with derivatives acting on $R^{4}$. The question is whether the whole series might be reproduced by summing loop diagrams of eleven-dimensional supergravity compactified on a circle.

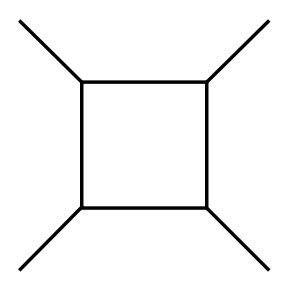

(a)

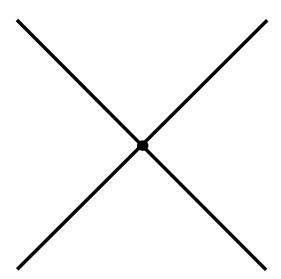

(b)

Figure 1: (a) The finite part of the one-loop diagram contributing to the $R^{4}$ term of compactified eleven-dimensional supergravity comes from the non-zero windings of the circulating particles. (b) The primitively divergent $R^{4}$ term that arises from the regularized zero winding sector is regularized to a specific finite value consistent with supersymmetry and various duality symmetries. 
We will restrict ourselves here to considering some simple multi-loop contributions that should reproduce the first few terms in the low-energy expansion of the string tree-level amplitude, (35). We have already seen that the constant term proportional to $\zeta(3)\left(R_{11}\right)^{-3}$ comes from the finite part of the one-loop diagram of figure 1(a). Figure 1(b) represents the counterterm for the primitive divergence of the one-loop diagram that is regularized to a finite value by the considerations outlined earlier.

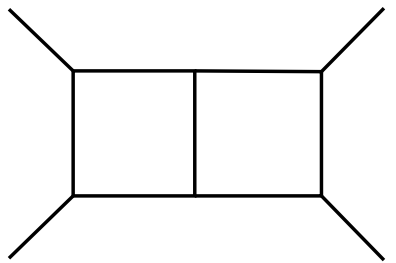

(a)

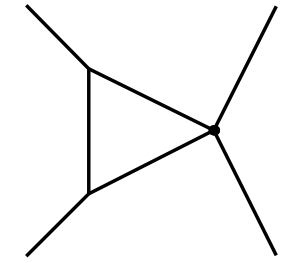

(b)

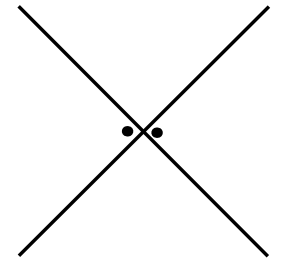

(c)

Figure 2: (a) A representation of the non-zero windings of the particles circulating in a two-loop contribution to four-graviton scattering. The dimension, (momentum $)^{20}$, of this finite contribution could give terms of the form $s^{3}$ in the ten-dimensional string tree-level amplitude. (b) A contribution in which one loop is replaced by the $R^{4}$ counterterm. has dimension (momentum) ${ }^{17}$ and could contribute terms of the form $s^{2}$ to the string tree-level amplitude. (c) A counterterm for the two-loop primitive divergence.

Just as with the one-loop diagrams, there are finite contributions from multi-loop diagrams which come from non-zero windings of the internal loops around the compact dimension(s). At each order there is also a new primitive divergence. For example, there are many two-loop diagrams that must be summed to give the complete amplitude. These have superficial degrees of divergence $\Lambda^{20}$, where $\Lambda$ is a momentum space cut-off and is $O\left(\kappa_{11}\right)^{4}$. However, we are discussing terms in the amplitude proportional to derivatives acting on $R^{4}$ so there are at least eight powers of the external momenta, reducing the naive divergence to $\Lambda^{12}$, or less (depending on the number of derivatives). This leads to new (two-loop) primitive divergences indicated by figure $2(\mathrm{c})$, where the two dots indicate the fact that this is a combination of two-loop counterterms. 
More generally, at $n$ loops there are primitive divergences of the form $\Lambda^{9 n-6} R^{4}$, where the powers of the cut-off $\Lambda$ may be substituted by powers of the external momenta. The ultra-violet divergences coming from the zero winding-number sector give rise to counterterms that are independent of $R_{11}$.

However, just as with the one-loop diagrams there will be finite terms arising from the effects of the internal propagators winding around the compact direction(s). These are collectively represented, in the case of the twoloop diagrams, by the ladder diagram in figure 2(a) which has dimension (momentum $)^{20}$. After accounting for the eight powers of the external momenta in the overall $R^{4}$, twelve powers of momenta remain that must be replaced by appropriate powers of the dimensional parameters, $R_{11}$ and $S, T, U$. This diagram may give a contribution to the tree-level string amplitude which is proportional to $\left(R_{11}\right)^{-3}$. In such a contribution there are nine powers of momentum left to be soaked up by a combination involving Mandelstam invariants. In the analogous expressions derived from string theory, such as (35), each Mandelstam invariant comes along with one power of $R_{11}^{-1}$. Thus, the nine powers of momenta should be associated with a linear combination of $S^{3} / R_{11}^{3}, T^{3} / R_{11}^{3}, S^{2} T / R_{11}^{3}$ and $T^{2} S / R_{11}^{3}$. We would like to identify this with the term in (35) that is cubic in the invariants. At $n$ loops the Feynman diagrams analogous to figure 2(a) can give finite contributions of the form $s^{3 n-3}$ in the expansion of the string tree amplitude (35).

In addition to these finite contributions there are also many other loop diagrams in which the counterterms are inserted as vertices. In fact, the diagram with the smallest primitive degree of divergence (other than the one-loop diagrams of figure 1) is the one shown in figure 2(b), in which the vertex indicated by a dot is the $R^{4}$ counterterm that was required to make sense of the one-loop diagram. Since all the particles of the supermultiplet circulate around the loop the supersymmetric partners of the $R^{4}$ vertices are also involved. These couple the two external gravitons to two $C^{(3)}$ 's, or to two gravitini. Figure 2(b) is meant to represent all diagrams of this order containing a single one-loop counterterm. These have dimension (momentum $)^{17}$ so that after allowing for the eight powers of external momenta in $R^{4}$ there are nine powers of momentum left to account for. These diagrams have not been explicitly evaluated but if it 
is assumed that they contribute to the string tree-level process there must be an overall factor of $e^{-2 \phi^{A}}=R_{11}^{-3}$, absorbing three of the nine momentum powers. Then dimensional counting implies that the the only possible finite function is quadratic in the invariants, $s, t, u \sim S / R_{11}, T / R_{11}, U / R_{11}$. This quadratic term ought to be precisely the first momentum-dependent correction to the $R^{4}$ term deduced by expanding the tree diagram. It should therefore turn out that an explicit calculation of figure 2(b) gives the finite contribution,

$$
\frac{2 \zeta(5)}{R_{11}^{3}} \tilde{K}\left(\frac{S^{2}+S T+T^{2}}{R_{11}^{2}}\right)
$$

where $\tilde{K}$ is the linearized approximation to $t_{8} t_{8} R^{4}$ and is eighth-order in the external momenta. Although the diagram in figure 2(b) has not been explicitly evaluated it is tempting to imagine that it has the form $\tilde{K} I(S, T, U)$, where $I$ is the expression for the loop amplitude of the same geometry in scalar field theory. This has a coefficient that includes a factor of $\zeta(5)$ just as there was a factor of $\zeta(3)$ in the finite part of the box diagram of figure 1(a). Verifying that the precise coefficient is the one in (36) would lend support to the suggestion that quantum corrections to compactified eleven-dimensional supergravity coincide with the string tree-level expressions at this order. In a similar manner the term with coefficient $\zeta(7)$ in (35) could arise from the diagam with two $R^{4}$ vertices and two propagators. However, this exhausts the contributions from one-loop diagrams with $R^{4}$ vertices and it is not at all clear how the terms of higher order in momenta in (35) would emerge in any systematic manner.

Of course, such diagrams also contribute to string loop effects. For example, there are the generalizations of the non-analytic one-loop massless threshold term, (45). In either of the type II string theories these terms can be written symbolically as

$$
\int \sqrt{g_{E}^{(10)}} s_{E}^{n} f_{n}\left(t_{E} / s_{E}\right) R^{4} d^{10} x=\int \sqrt{g^{(10)}} e^{\frac{n-1}{2} \phi^{A}} s^{n} f_{n}(t / s) R^{4} d^{10} x,
$$

where $g_{E}$ is the type II string metric in the Einstein frame. The function $f_{n}(t / s)$ involves up to $n$ powers of $t / s$ as well as factors of $\log (t /(s+t))$ and $\log (s /(s+t))$. The $n=1$ case is given implicitly by (45). Since these terms arise in string perturbation theory, which is an expansion in $e^{2 \phi}$, it must be 
that $n=4 m+1$, where $m$ is an integer. In the Einstein frame these terms are independent of the dilaton so they are inert under the action of $S L(2, Z)$ in the IIB theory. They are therefore not constrained by S-duality. In the IIA theory such terms are directly related to analogous thresholds in genus- $(m+1)$ multiloop eleven-dimensional supergravity amplitudes. These Feynman diagrams are dimensionally of the form (momentum) ${ }^{9 m+3} R^{4}$. After compactification their contribution to the genus- $(m+1)$ string amplitude has a factor of $e^{2 m \phi^{A}}=$ $R_{11}^{3 m}$ from powers of the coupling. The total momentum dimension of this contribution is then written as $R_{11}^{3 m}$ (momentum $)^{12 m+3} R^{4}$, which must therefore be made up of terms of the form $e^{2 m \phi^{A}} s^{4 m+1} f_{n}(t / s) R^{4}$ in the string coordinates, using the fact that $s=S / R_{11}$. This agrees in structure with the terms in (37) if the function $f$ contains the appropriate logarithmic terms.

There are many complications in understanding in detail the systematics of the correspondence between the higher-loop supergravity diagrams and string diagrams. Whereas the $R^{4}$ and related terms of the same dimension are integrals over half the superspace, terms with more derivatives are integrals over a higher fraction of the superspace. Each power of momentum is equivalent to two powers of $\theta$ so that terms with less than eight powers of momentum acting on $R^{4}$ should be protected and may be determined in this manner. This would include the terms up to the $\zeta(3)^{2}$ term in (35) and possibly also the $\zeta(7)$ term. Whether it is possible to go beyond this and relate terms in string perturbation theory at higher order in the momentum expansion to eleven-dimensional supergravity is much less obvious.

\section{Acknowledgements:}

I am grateful to Nati Seiberg, Paul Howe and Adam Schwimmer, as well as participants at the Aspen Center for Physics Workshop on String Dualities in the Summer of 1997, for useful discussions.

\section{A Momentum dependence of loop amplitude}

The momentum dependence of the four-graviton amplitude is contained in the functions $I_{n}(S, T)(n=2, \cdots, \infty)$ in (19). This will be a homogeneous poly- 
nomial of degree $n$. There is no $n=1$ term after adding the contributions of $I(S, U)$ and $I(T, U)$ since there is no nonvanishing linear symmetric combination. The term with zero Kaluza-Klein momenta is given by

$$
I^{0}(S, T)=\frac{1}{\mathcal{V}_{2}} \int \prod_{r=1}^{4} d \tau_{r}(\tau)^{-9 / 2}\left(e^{\left(S \tau_{1} \tau_{3}+T \tau_{2} \tau_{4}\right) / \tau}-1\right),
$$

where the -1 accounts for the fact that the constant terms are contained in $I_{0}$. The variables $\tau_{r}$ may be redefined by

$$
\omega_{1}=\frac{\tau_{1}}{\tau}, \quad \omega_{2}=\frac{1}{\tau}\left(\tau_{1}+\tau_{2}\right), \quad \omega_{3}=\frac{1}{\tau}\left(\tau_{1}+\tau_{2}+\tau_{3}\right), \quad \tau=\sum_{r=1}^{4} \tau_{r},
$$

which are to be integrated over the region $0 \leq \omega_{1} \leq \omega_{2} \leq \omega_{3} \leq 1$ so that

$$
I^{0}(S, T)=\frac{1}{\mathcal{V}_{2}} \int_{0}^{\infty} d \tau \int \prod_{r=1}^{3} d \omega_{r}(\tau)^{-3 / 2}\left(e^{-Q\left(S, T ; \omega_{r}\right) \tau}-1\right)
$$

where

$$
Q\left(S, T ; \omega_{r}\right)=-S \omega_{1}\left(\omega_{3}-\omega_{2}\right)-T\left(\omega_{2}-\omega_{1}\right)\left(1-\omega_{3}\right) .
$$

The integral in (40) is simply evaluated by noting that $\partial I^{0} / \partial Q=$ $\sqrt{\pi} \int \prod d \omega_{r} Q^{-\frac{1}{2}}$ so that

$$
I^{0}(S, T) \equiv 2 \sqrt{\pi} \mathcal{S}^{\frac{1}{2}}=2 \sqrt{\pi} \int \prod d \omega_{r}\left(-Q\left(S, T ; \omega_{r}\right)\right)^{\frac{1}{2}}
$$

where, more generally, $\mathcal{S}^{n}$ is defined by

$$
\mathcal{S}^{n}=\int \prod d \omega_{r}\left(-Q\left(S, T ; \omega_{r}\right)\right)^{n}
$$

Similarly, $\mathcal{T}^{n}$ and $\mathcal{U}^{n}$ will be defined by cyclically permuting $S, T$ and $U$ in the function $Q$.

This non-analytic term (42) in nine-dimensional M-theory translates into a similar term in either nine-dimensional string theory (as was also noticed in [9]) making use of the familiar relation, by $g^{(9)}=R_{11} G^{(9)}$, between the ninedimensional type II metric, $g_{\mu \nu}^{(9)}$, and the M-theory metric, $G^{(9)}$, compactified on $T^{2}$.

Using the relation between the M-theory and string theory Mandelstam invariants (33) it follows that

$2 \sqrt{\pi} \int \sqrt{G^{(9)}} R^{4}\left(\mathcal{S}^{\frac{1}{2}}+\mathcal{T}^{\frac{1}{2}}+\mathcal{U}^{\frac{1}{2}}\right) d^{9} x=2 \sqrt{\pi} \int \sqrt{g^{(9)}} R^{4}\left(\mathcal{S}_{s}^{\frac{1}{2}}+\mathcal{T}_{s}^{\frac{1}{2}}+\mathcal{U}_{s}^{\frac{1}{2}}\right) d^{9} x$, 
where the expressions $\mathcal{S}_{s}, \mathcal{T}_{s}$ and $\mathcal{U}_{s}$ are defined in terms of the Mandelstam invariants of string theory. The analogous expression in the limit of decompactification, $R_{10} \rightarrow \infty$, is of the form,

$$
\begin{aligned}
& \int \sqrt{G^{(10)}} R^{4}(\mathcal{S} \ln \mathcal{S}+\mathcal{T} \ln \mathcal{T}+\mathcal{U} \ln \mathcal{U}) d^{10} x \\
& =\int \sqrt{g^{A(10)}} R^{4}\left(\mathcal{S}_{s} \ln \mathcal{S}_{s}+\mathcal{T}_{s} \ln \mathcal{T}_{s}+\mathcal{U}_{s} \ln \mathcal{U}_{s}\right) d^{10} x
\end{aligned}
$$

The scale of the logarithms cancels out after using the condition $s+t+u=0$. Both (44) and (45) have imaginary parts corresponding to the massless normal thresholds determined by unitarity. However, the real parts, which might have given rise to arbitrary constants, are here fixed to precise values.

The terms $I_{n}(S, T)$ in (19) are given by by expanding the exponential in (14) giving,

$$
\begin{aligned}
I_{n}(S, T) & =\int_{0}^{\infty} \frac{d \tau}{\tau^{\frac{3}{2}-n}} \int \prod_{r=1}^{3} d \omega_{r} \sum_{\left(l_{1}, l_{2}\right) \neq(0,0)} e^{-G^{I J} l_{I} l_{J} \tau} \frac{\left(-Q\left(S, T ; \omega_{r}\right)\right)^{n}}{n !} \\
& =\Gamma\left(n-\frac{1}{2}\right) \zeta\left(n-\frac{1}{2}\right) E_{n-\frac{1}{2}}(\Omega, \bar{\Omega}) \int \prod_{r=1}^{3} d \omega_{r} \frac{\left(-Q\left(S, T ; \omega_{r}\right)\right)^{n}}{n !}
\end{aligned}
$$

Putting all the terms together the complete expression for the $R^{4}$ term in the one-loop effective action for eleven-dimensional supergravity on $T^{2}$ is given by (13) with the function $h$ defined by the amplitude $A_{4}$ in (9) where

$$
\begin{aligned}
& I(S, T)+I(T, U)+I(U, S)= \\
& I_{0}+2 \sqrt{\pi} \mathcal{W}^{\frac{1}{2}}+\sum_{n=2}^{\infty} \frac{\mathcal{W}^{n} \mathcal{V}_{2}^{n-\frac{3}{2}}}{n !}\left[\Gamma\left(n-\frac{1}{2}\right) \zeta(2 n-1)\left(\frac{R_{10}}{R_{11}}\right)^{n-\frac{1}{2}}\right. \\
& \left.+\sqrt{\pi} \Gamma(n-1) \zeta(2 n-2)\left(\frac{R_{10}}{R_{11}}\right)^{\frac{3}{2}-n}\right]+ \text { non - perturbative terms, }
\end{aligned}
$$

and

$$
\mathcal{W}^{n}=\mathcal{S}^{n}+\mathcal{T}^{n}+\mathcal{U}^{n} .
$$

The $S, T$ or $U$-dependent terms in (47) exponentiate in the form $e^{S \mathcal{V}_{2}}$ which vanishes in the infinite volume limit, $\mathcal{V}_{2} \rightarrow \infty$.

It follows from (47) that the expression for the nine-dimensional effective action arising from the one-loop supergravity amplitude can be written in IIA 
coordinates as

$$
\begin{gathered}
\int \sqrt{g^{A(9)}} r^{A} R^{4}\left[I_{0}+\frac{1}{r_{A}} \mathcal{W}_{s}^{\frac{1}{2}}+\sum_{n=2}^{\infty}\left(\frac{1}{r_{A}^{2}} \frac{r_{A}^{2 n} \mathcal{W}_{s}^{n}}{n !}+e^{-2 \phi^{A}} \frac{e^{2 n \phi^{A}} \mathcal{W}_{s}^{n}}{n !}\right)\right] d^{9} x \\
+ \text { non - perturbative terms }
\end{gathered}
$$

where

$$
\mathcal{W}_{s}^{n}=\mathcal{S}_{s}^{n}+\mathcal{T}_{s}^{n}+\mathcal{U}_{s}^{n},
$$

and $I_{0}$ can be reexpressed in type IIA parameters, $r_{A}$ and $\phi^{A}$. The first sum in round parentheses gives terms of the form $e^{r_{A}^{2} s}$ which are exponentially suppressed in the ten-dimensional limit, $r_{A} \rightarrow \infty$. The ten-dimensional limit in the IIA theory is therefore given by a power series in $e^{2 \phi^{A}} s$ or, for constant dilaton, by a series of powers of $g_{A}^{2} s$ where $g_{A}=e^{\phi^{A}}$. Each power, $n$, should presumably be identified with a contribution from a string loop amplitude of genus $n+1$. This suggests that the leading low-momentum term at genus $n$ of the IIA theory should be of order $\left(g_{A}^{2} k^{2}\right)^{n-1}$, where $g_{A}=e^{\phi^{A}}$ for constant $\phi^{A}$ (and $k^{2}$ represents some combination of $s, t$ and $u$ ).

In the IIB coordinates the action becomes

$$
\begin{gathered}
\sum_{n} \int \sqrt{g^{B(9)}} r^{B} R^{4}\left[I_{0}+\frac{1}{r^{B}} \mathcal{W}_{s}^{\frac{1}{2}}+\sum_{n=2}^{\infty}\left(\frac{1}{n !} \frac{\mathcal{W}_{s}^{n}}{r_{B}^{2 n}}+\frac{e^{-2 \phi^{B}}}{n !} \frac{\mathcal{W}_{s}^{n} e^{2 n \phi^{B}}}{r_{B}^{2 n}}\right)\right] d^{9} x \\
+ \text { non - perturbative terms. }
\end{gathered}
$$

All the momentum-dependent terms in this expression vanish in the limit $r_{B} \rightarrow \infty$ with fixed coupling $e^{\phi^{B}}$. This means that these terms do not contribute to the loop amplitudes of the ten-dimensional type IIB theory. However, the four-graviton loop amplitudes are identical in the IIA and IIB theories as a consequence of T-duality. This means that there must be terms in an expansion in powers of $e^{2 \phi^{B}} s=g_{B}^{2} s$ that have another eleven-dimensional origin. For example, such terms can arise from eleven-dimensional multi-loop diagrams compactified on a torus.

\section{References}

[1] E. Cremmer, B. Julia and J. Scherk, Supergravity theory in eleven dimensions, Phys. Lett. 76B (1978) 409. 
[2] M.J. Duff, J.T. Liu and R. Minasian, Eleven-dimensional origin of string-string duality: a one loop test, hep-th/9506126, Nucl. Phys. 452B (1995)261.

[3] C. Vafa and E. Witten, A one loop test of string duality, hep-th/9505053, Nucl. Phys. B447 (1995)261.

[4] Blum and J. Harvey, Anomaly inflow for gauge defects, hep-th/9310035; Nucl. Phys. B416 (1994) 119.

[5] P. Aspinwall, Some Relationships Between Dualities in String Theory, in Proceedings of 'S-duality and mirror symmetry', Trieste 1995, hepth/9508154, Nucl. Phys. Proc. 46 (1996) 30.

[6] J.H. Schwarz, Lectures on Superstring and M-theory dualities, hepth/9607201; J.H. Schwarz, An Sl(2,Z) multiplet of type IIb superstrings, hep-th/9508143 Phys. Lett. 360B (1995) 13.

[7] M.B. Green, M. Gutperle and P. Vanhove, One loop in eleven dimensions, hep-th/9706175, Phys. Lett. 409B (1997)177.

[8] M.B. Green and M. Gutperle, Effects of D-instantons, hep-th/9701093, Nucl. Phys. B498 (1997)195.

[9] J.G. Russo and A.A. Tseytlin, One loop four graviton amplitude in eleven-dimensional supergravity hep-th/9707134.

[10] M.B. Green, M. Gutperle and H. Kwon, Sixteen-fermion and related terms in $M$ theory on $T^{2}$, hep-th/9710151.

[11] M.B. Green and Michael Gutperle, D-particle bound states and the Dinstanton measure hep-th/9711107.

[12] E. Witten, Bound States of Strings and p-branes, Nucl. Phys. B460 (1996) 335.

[13] S. Sethi and M. Stern, D-brane bound states redux, hep-th/9705046.

[14] P. Yi,Witten index and threshold bound states of D-branes, hepth/9704098. 
[15] M.B. Green and J.H. Schwarz, Supersymmetric dual string theory (III). Loops and renormalization, Nucl. Phys. 198B (1982) 441.

[16] M.T. Grisaru, A.E.M Van de Ven and D. Zanon, Two-dimensional supersymmetric sigma models on Ricci flat Kähler manifolds are not finite, Nucl. Phys. B277 (1986) 388; Four loop divergences for the N=1 supersymmetric nonlinear sigma model in two-dimensions, Nucl. Phys. B277 (1986) 409.

[17] D.J. Gross and E. Witten, Superstring modifications of Einstein's equations, Nucl. Phys. B277 (1986) 1.

[18] M.B. Green and J.H. Schwarz, Supersymmetrical string theories, Phys. Lett. 109B (1982) 444.

[19] A. Terras, Harmonic Analysis on Symmetric Spaces and Applications, vol. I, Springer-Verlag (1985).

[20] I. Antoniadis, B. Pioline and T.R. Taylor, Calculable $e^{1 / \lambda}$ effects, hepth/9707222.

[21] N. Berkovits, Construction of $R^{4}$ terms in $N=2 D=8$ superspace, hepth/9709116.

[22] R. Jengo and C.-J. Zhu, Two loop computation of the four particle amplitude in heterotic string theory, Phys. Lett. 212B (1988) 313.

[23] T. Banks, W. Fischler, S.H. Shenker and L. Susskind, M-Theory as a matrix model: a conjecture, hep-th/9610043, Phys. Rev. D55 (1997) 5112 .

[24] N. Nekrasov and A. Lawrence, Instanton sums and five dimensional gauge theories, hep-th/9706025.

[25] M.B. Green and P. Vanhove, D-instantons, strings and M-theory, hepth/9704145, Phys. Lett. 408B (1997)122.

[26] E. Kiritsis and B. Pioline, On $R^{4}$ threshold corrections in IIB string theory and $(p, q)$ string instantons, hep-th/9707018. 
[27] C. Bachas, C. Fabre, E. Kiritsis, N.A. Obers and P. Vanhove, Heterotic/type I duality and D-brane instantons, hep-th/9707126.

E. Kiritsis and N.A. Obers, Heterotic/type-I duality in $D<10$ dimensions, threshold corrections and D-instantons, hep-th/9709058.

[28] C. Bachas, Heterotic versus type I, Talk at STRINGS'97 (Amsterdam, June 16-21 1997) and HEP-97 (Jerusalem, August 19-26 1997), hepth/9710102.

[29] J.H. Schwarz and P.C. West, Symmetries and transformations of chiral N=2 D=10 supergravity, Phys. Lett. 126B (1983) 301.

[30] J.H. Schwarz, Covariant field equations of chiral N=2 D=10 supergravity, Nucl. Phys. B226 (1983) 269.

[31] P.S. Howe and P.C. West, The complete N=2 D=10 supergravity, Nucl. Phys. B238 (1984) 181.

[32] D.J. Gross and J.H. Sloan, The quartic effective action for the heterotic string, Nucl. Phys. 291B (1987) 41.

[33] A. Kehagias and H. Partouche, The exact quartic effective action for the type IIB superstring, hep-th/9710023.

[34] N. Ishibashi, H. Kawai and Y. Kitazawa and A. Tsuchiya, A large $N$ reduced model as superstring, hep-th/9612115; Nucl.Phys.B498:467491,1997. 